\title{
Modeling, Calibration, and Verification of a Fission Chamber for ACRR Experimenters ${ }^{\mathrm{a}}$
}

\author{
Jonathan Coburn ${ }^{1}$, S. Michael Luker ${ }^{2, b}$, Edward J. Parma ${ }^{3}$, and K. Russell DePriest ${ }^{3}$ \\ ${ }^{1}$ Graduate Student Intern (North Carolina State University), Applied Nuclear Technologies, Sandia \\ National Laboratories, Albuquerque, New Mexico, USA \\ ${ }^{2}$ Senior R\&D Scientist/Engineer, Applied Nuclear Technologies, Sandia National Laboratories, \\ Albuquerque, New Mexico, USA \\ ${ }^{3}$ Principal R\&D Scientist/Engineer, Applied Nuclear Technologies, Sandia National Laboratories, \\ Albuquerque, New Mexico, USA
}

\begin{abstract}
When performing research at a reactor facility, experimenters often need to determine the neutron fluence achieved during an operation. Facilities typically provide guidance in the form of neutron fluence per megajoule (MJ) or through passive dosimetry results. After experiment completion, there is sometimes a delay of several days (or weeks) before the passive dosimetry results are available. In the interim, an experimenter does not have confirmation that the desired irradiation levels were reached. Active dosimetry may provide an estimate of neutron fluxes, but few active detectors are available that have been calibrated to measure neutron fluxes obtained inside the Annular Core Research Reactor (ACRR) central cavity environment. For past experiments at the ACRR, the neutron fluence was calculated by integrating the response of a fission chamber rate detection signal and then normalizing this integral to fluence determined from passive dosimetry. An alternative method of directly measuring neutron flux is desired; the new methodology described provides a complete neutron flux profile after a reactor pulse, utilizing fission chamber physics in combination with a compensating ion chamber to extract and convert a current signal to neutron flux as a function of time.
\end{abstract}

\section{Introduction and Theory}

\subsection{Fission Chamber Basics}

A fission chamber is essentially an ionization chamber coated with a small amount of fissile material, such as ${ }^{235} \mathrm{U}$, along the wall. Figure 1 shows a cross sectional view of a typical fission chamber housing. The chamber is filled with an inert noble gas such as argon. The outer cylinder and the inner wire have

\footnotetext{
${ }^{a}$ Work supported by the United States Department of Energy at Sandia National Laboratories. Sandia National Laboratories is a multi-program laboratory managed and operated by Sandia Corporation, a wholly owned subsidiary of Lockheed Martin Corporation, for the U.S. Department of Energy's National Nuclear Security Administration under contract DE-AC0494AL85000.

b Corresponding author: smluker@sandia.gov
}

This is an Open Access article distributed under the terms of the Creative Commons Attribution License 2.0, which permits unrestricted use, distribution, and reproduction in any medium, provided the original work is properly cited. 


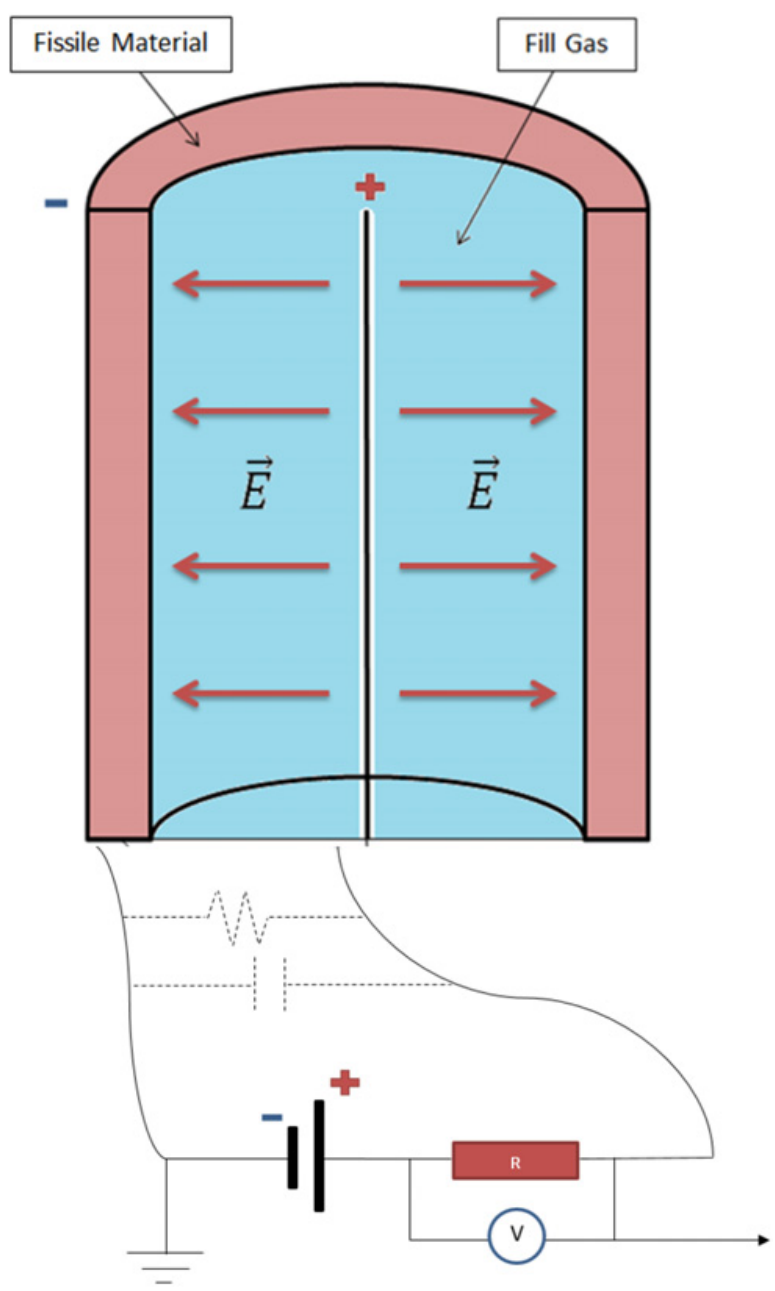

Figure 1. Cross section view (and schematic) of a typical fission chamber.

a voltage applied between them creating a radially symmetrical electric field between the cathode and the anode of the chamber.

A fission chamber works to detect neutrons as follows (illustrated in Fig. 2):

(1) Incident neutron is absorbed with a fissile nucleus and causes fission.

(2) The fissile nucleus splits into two fission fragments. Due to conservation of momentum, the fission fragments travel in opposite directions. One of the fission fragments will travel into the gas region of the detector.

(3) The fission fragments have a high linear energy transfer, and will deposit most if not all of their energy in the fill gas by ionizing the gaseous atoms.

(4) Due to the applied voltage, free electrons are collected on the inner anode wire, producing a current that is output by the fission chamber.

In general, the amount of current generated by the fission chamber by these physical processes is proportional to the rate of fission events produced in the detector. Since the current is proportional 


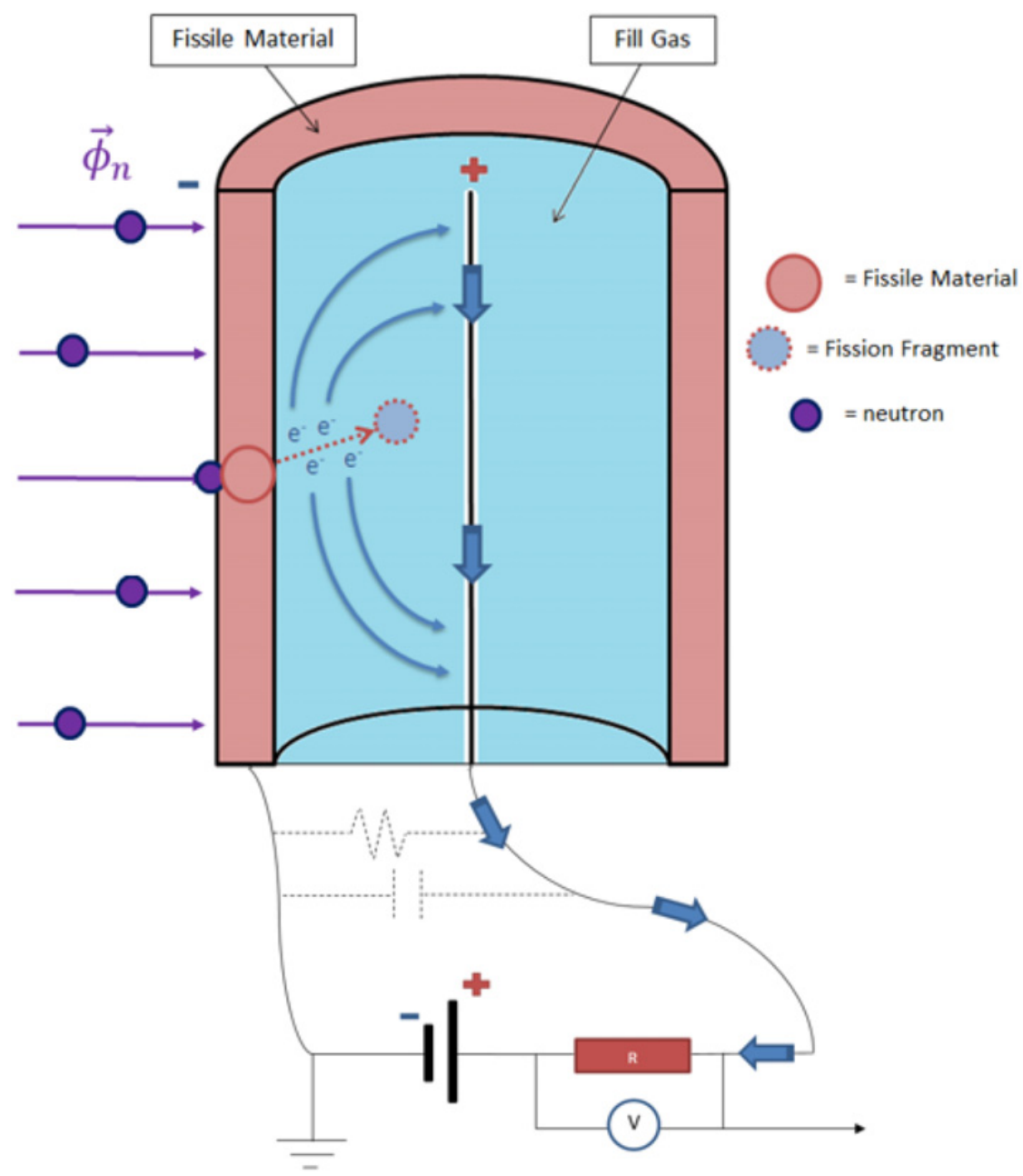

Figure 2. Illustration of the fission chamber physical processes.

to the fission rate, the current is also proportional to the incident neutron flux [1]. The magnitude of this proportionality is largely dependent on two factors: (1) the spectrum-averaged fission cross section for the fissile materials for the incident neutrons and (2) the characteristic energy required to ionize atoms of the fill gas. Thus, for a given neutron environment, the relationship between net neutron fission chamber current $I_{n}(t)$ and neutron flux is given by Eq. (1):

$$
I_{n}(t)=F \cdot A \cdot x \int \varphi_{n}(E, t) \cdot \Sigma_{f}(E) d E
$$

where $F$ is a chamber dependent constant (related to the Coulombs of charge produced per fission within the detector), $A$ is the active detector area, $\mathrm{x}$ is the fissile material thickness, $\varphi_{n}(E, t)$ is the neutron flux as a function of neutron energy and time, and $\Sigma_{f}(E)$ is the macroscopic fission cross section of the uranium oxide coating in $\mathrm{cm}^{-1}$ as a function of neutron energy. A useful relationship between the current and the neutron flux is obtained by integrating and rearranging Eq. (1), resulting in Eq. (2):

$$
\overline{\varphi_{n}}(t)=\frac{I_{n}(t)}{F \cdot A \cdot x \cdot \overline{\Sigma_{f}}}
$$


Where $F, A$, and $\mathrm{x}$ are aspects of a given fission chamber and $\overline{\Sigma_{f}}$ is dependent upon the neutron environment. Equation (2) assumes that the flux can be split into separate functions: $\varphi_{n}(E, t)=$ $\varphi_{n}(t) f(E)$, where $\mathrm{f}$ is the normalized neutron spectrum. The method outlined attempts to utilize only the current produced by neutrons inducing fission in the fission chamber, but the fission chamber is also sensitive to ionization caused by gamma radiation present in the reactor environment. The interaction of prompt fission gammas, on the order of $\mathrm{MeV}$, will be dominated by Compton scattering in the fill gas [5]. In order to correct for this additional current, a compensating ion chamber can be fielded with the fission chamber. The gamma ionization contribution to the fission chamber is removed using Equation (3), provided that both chambers have the same gamma signal or that any difference in response can be quantified:

$$
I_{n}(t)=I_{\text {total }}(t)-I_{\gamma}(t)
$$

where $I_{\gamma}(t)$ is the ionization current in the chamber produced by gamma radiation. It is assumed that gamma signals from neutron activation is negligible. If fielded in a characterized reactor environment where the neutron spectrum is well established, this compensated method employing the fission chamber will successfully provide a time-dependent neutron flux profile from the detector signal.

\subsection{Evaluation of Calibration Technique in ACRR LB36 Environment}

In order to utilize this fission chamber calibration technique in experiments, the fission chamber ( $F$ and $A$ ) and reactor environment $\left(\overline{\Sigma_{f}}\right)$ factors in Eq. (2) must be determined. For a well-characterized neutron environment, the value of $\overline{\Sigma_{f}}$ is easily calculated. The value of $A$ is readily determined by examining the appropriate schematics of the fission chamber design. This work documents the experimental determination of a miniature Photonis fission chamber in the ACRR lead-boron 36" bucket (LB36) environment and provides validation of the experimental technique through other dosimetry methods. The specific detector used in the experiment is a Photonis fission chamber / compensating ion chamber (CIC) pair, model numbers CFUF53/SA-U5 and CRGF10/SA. The fission chamber contains $39 \mu \mathrm{g}$ of uranium, in oxide form, with $92.1 \mathrm{w} / \mathrm{o} \mathrm{U}^{235}, 6.6 \mathrm{w} / \mathrm{o} \mathrm{U}^{238}$, and $1.3 \mathrm{w} / \mathrm{o} \mathrm{U}^{234}$. The uranium coating, $\mathrm{x}$, is approximately $0.0102 \mu \mathrm{m}$. Both chambers have similar dimensions with an active region of $3.987 \mathrm{~cm}^{2}$, an active length of $27 \mathrm{~mm}$, are filled with argon gas, and are operated at 150 volts.

The value of $F$ can be obtained by integrating Eq. (2) with respect to time and rearranging:

$$
F=\frac{Q}{\overline{\Phi_{n}} \cdot A \cdot x \cdot \overline{\Sigma_{f}}}
$$

where $Q$ (Coulombs) is the integrated charge over the time span of the neutron flux event and $\overline{\Phi_{n}}$ $\left(\mathrm{n} / \mathrm{cm}^{2}\right)$ is the total neutron fluence over the time of the event. The value of $\mathrm{Q}$ for an event is obtained by integrating the signal current $\left(I_{n}(t)\right)$, but the value of $\overline{\Phi_{n}}$ requires additional passive dosimetry. Initially, the average neutron fluence was estimated using a detailed ACRR model in MCNP [2], based off of SAND report values for the LB36 environment $[3,4]$. The estimate of the neutron fluence was refined and/or corrected through the use of appropriate dosimetry monitor foils as shown in Fig. 3.

The fission chamber/ion chamber pair was fielded in the LB36 environment at the core centerline as illustrated in Fig. 4. The LB36 environment is an epithermal neutron environment with a reduced gamma radiation environment (compared to the ACRR free-field central cavity environment). The experimental setup included a second LND-brand ion chamber, a diamond photo-conducting detector (PCD), boron and silicon calorimeters, nickel dosimetry foils, and sulfur pellets. The setup was fielded during multiple reactor operations (reactor pulses and low power steady-state runs). The entire package of passive dosimeters was retrieved between reactor operations, but the fission chamber and other active detectors were not disturbed while the dosimetry retrieval operations occurred. 


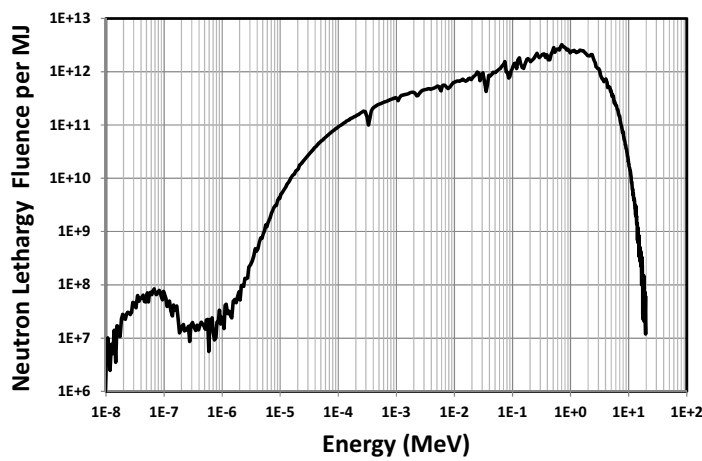

Figure 3. LB36 neutron spectrum.

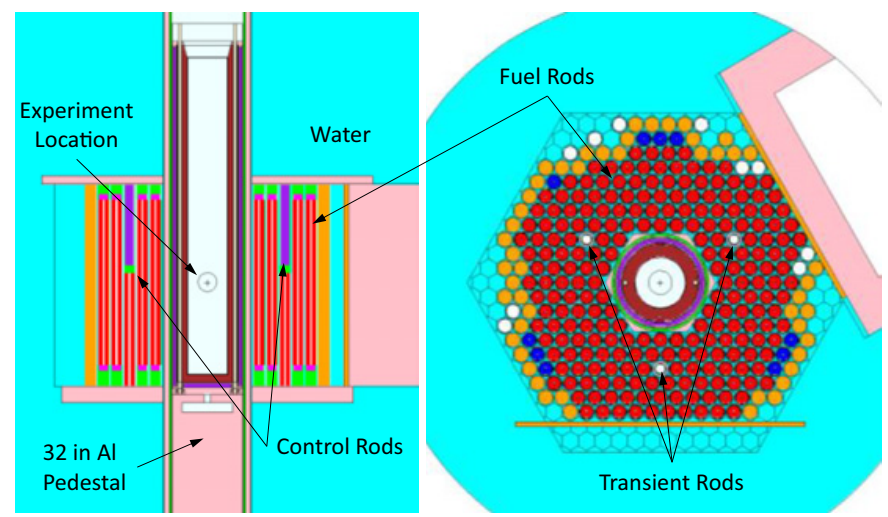

Figure 4. Experiment location in reactor core at fuel-centerline.

The following outline was the general procedure for analysis of the fission chamber in the reactor environment.

(1) Perform reactor operation (pulse or steady-state) and obtain real-time data for $I_{n}(t)$.

(2) Integrate $I_{n}(t)$ over the data set to obtain a value for $Q$.

(3) Analyze passive dosimeters to obtain $\overline{\Phi_{n}}$ from each dosimetry method.

(4) Relate $\overline{\Phi_{n}}$ to the number of fissions that occurred during the reactor operation using Eq. (4).

(5) Calculate $F$ based on $Q$ and the number of fissions.

Each dosimetry method produced a different value of $F$. It was observed that the impact of having different detector locations was negligible. The values of $F$ for each dosimetry technique were examined and compared with MCNP-predicted results [6].

\subsection{LND Ion Chamber Substitute as Compensating Ion Chamber}

Due to some anomalies with the Photonis CIC, it was necessary to utilize another ion chamber during the experiment. In previous low power, steady-state experiments, the fission chamber's compensating ion chamber developed experimental anomalies, including phenomenon such as producing a gamma current signal greater than the fission chamber and producing a signal that is not responsive to a low gamma flux. During the experiment series, it was again concluded that the CIC was malfunctioning. 
Table 1. Experimental Conditions and Constants.

\begin{tabular}{|c|c|c|c|c|}
\hline Reactor Operations & Type & Reported Energy [MJ] & Calculated Energy [MJ] & Uncertainty [\%] \\
\hline Run 1, Shot\#10715 & Pulse & 36 & 53.4 & 5.8 \\
\hline Run 2, Shot\#10716 & Pulse & 47.3 & 55.1 & 5.8 \\
\hline Run 3, Shot\# 10722 & Pulse & 50 & 52.9 & 5.8 \\
\hline Run 4, Shot\# 10721 & Pulse & 98 & 100.0 & 5.8 \\
\hline Run 5, SS \#10718 & Steady-state & 8.5 & 9.62 & 5.81 \\
\hline Run 6, SS\# 10719 & Steady-state & 22.2 & 19.6 & 5.81 \\
\hline Run 7, SS\# 10720 & Steady-state & 88.1 & 90.34 & 5.81 \\
\hline \multicolumn{2}{|c|}{ MCNP Fissions/MJ Constant: } & \multicolumn{2}{|r|}{$3.04 * 10^{6}[$ Fissions/MJ] } & \\
\hline \multirow{2}{*}{\multicolumn{2}{|c|}{$\mathbf{A}_{\mathrm{FC}} / \mathbf{A}_{\mathrm{LND}}$ Ratio: }} & \multicolumn{2}{|r|}{0.0977} & \\
\hline & $\mathbf{G}_{\mathrm{FC}} / \mathrm{G}_{\mathrm{LND}}$ Ratio: & \multicolumn{2}{|r|}{0.844} & \\
\hline \multicolumn{2}{|c|}{$\overline{\boldsymbol{\Sigma}_{f}}$ for LB36 Environment } & \multicolumn{2}{|r|}{$0.0679\left[\mathrm{~cm}^{-1}\right]$} & \\
\hline
\end{tabular}

An experiment at Sandia's Gamma Irradiation Facility was designed to investigate the fission chamber and CIC response in a gamma-only environment. An LND gamma ionization chamber, model number 52139, was included as a backup ion chamber, nitrogen filled at 68 Torr with an active length of $50 \mathrm{~mm}$. Results were poor for the paired CIC for this experiment, indicating that it was malfunctioning. However, experiment results were obtained such that the LND chamber could be utilized with a new compensation technique for the fission chamber. Ion chamber signals that see the same gamma spectrum can be related based on the detector area, $A$, and the fill gas ionization potential, $G$, as seen in Eq. (5):

$$
\varphi_{\gamma}(t)=\frac{I_{1}(t)}{G_{1} \cdot A_{1}}=\frac{I_{2}(t)}{G_{2} \cdot A_{2}} .
$$

Thus, the current signals for the fission chamber and some other ion chamber such as the LND ion chamber can be related by Eq. (6) as long as the $G$ and $A$ ratios are known.

$$
I_{\gamma-F C}(t)=\left(\frac{G_{F C}}{G_{L N D}}\right)\left(\frac{A_{F C}}{A_{L N D}}\right) I_{L N D}(t) .
$$

In this experiment, both the LND and fission chambers were fielded in the Gamma Irradiation Facility for a given amount of time. With the A ratio known and simultaneous current signals obtained, the $\mathrm{G}$ ratio for the two detectors was calculated utilizing Eq. (6).

\section{Experimental Results}

The fielding experiment of the fission chamber in the LB36 ACRR environment consisted of four pulse runs and three steady-state runs, each at varying energy levels. Each run and its associated energy output is given in Table 1. Also included are important constants used in calculating the conversion factor $F . \overline{\Sigma_{f}}$ for the fission chamber uranium coating was calculated using MCNP5 v1.60 for the LB36 environment. The conversion factor was estimated using MCNP and measured using nickel and sulfur dosimetry for all seven runs. MCNP results are rescaled using the energy observed in each reactor run, while dosimetry results are calculated independently. Sample data from pulse run 3 can be seen in Fig. 5 .

\subsection{Pulse Results}

ACRR pulsed experiment results were obtained for each run. Current signals were collected from the fission chamber, LND ion chamber, and the CIC. Although the CIC showed no response for steady-state runs, it appeared to respond as expected during pulsed operation. Thus, the fission chamber conversion 


\section{$15^{\text {th }}$ ISRD}

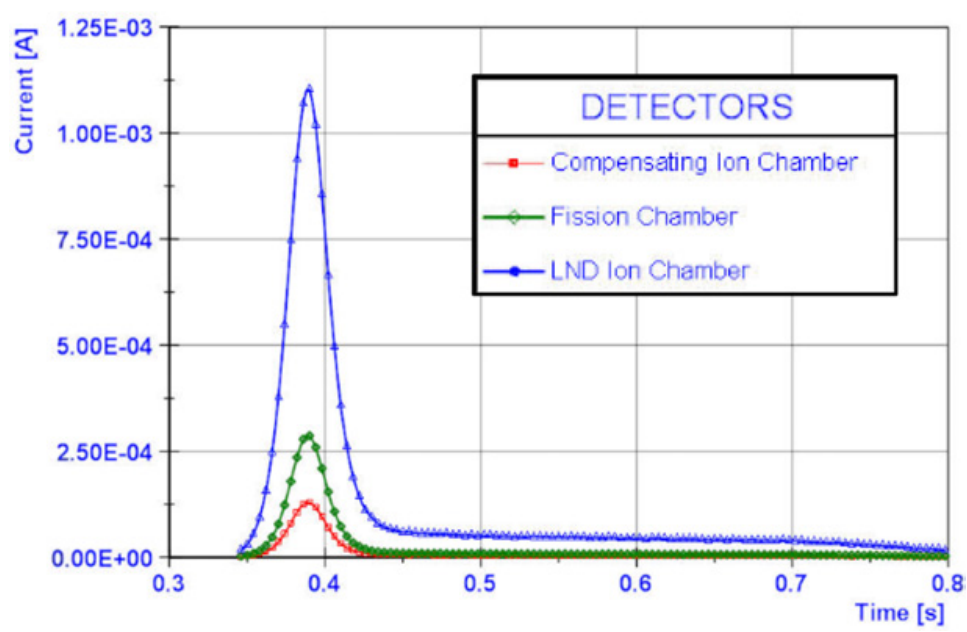

Figure 5. Sample detector data for run \#3 (50 MJ pulse).

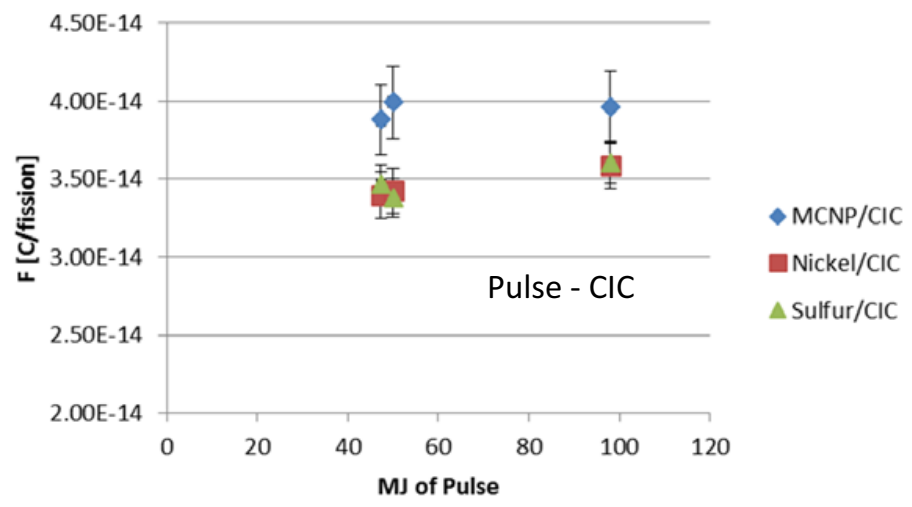

Figure 6. Pulse results for $F$ calculated from CIC signal.

factor F was calculated using the CIC current signal, combined with data from nickel dosimetry, sulfur pellets, and MCNP estimations. All results are tallied in Fig. 6.

The CIC current signal data was clipped for the $36 \mathrm{MJ}$ pulse, and was not usable. The values for $F$ are nearly identical for the nickel and sulfur dosimetry data; the MCNP data appears to overestimate the value for $F$, likely due to an underestimation in the number of fissions that occur within the fission chamber. This overestimation was only seen for the pulse data.

\subsection{Steady-state Results}

ACRR steady-state experiment results were obtained for all runs. Current signals were collected from the fission chamber and LND, while the CIC gave unresponsive results. The fission chamber conversion factor F was calculated using the LND current signal, combined with data from nickel dosimetry, sulfur pellets, and MCNP estimations. All results are tallied in Fig. 7. It is important to note that for low flux, steady-state runs, it is necessary to subtract the background counts for both the LND chamber and the fission chamber. Although background can be neglected for pulse runs, steady-state runs occurred over a much longer timeframe ( $\sim 9$ minutes) and gave much smaller current signals. Thus background 


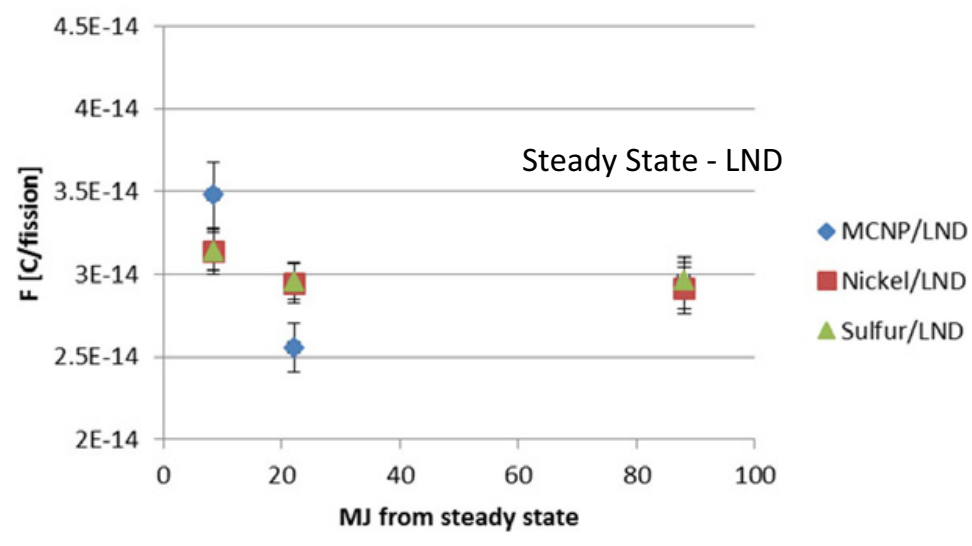

Figure 7. Steady-state results for $F$ calculated from LND signal.

counts from both the reactor central cavity and from activity within the fission chamber contributed significantly to the total current signals at low reactor power levels.

The results show good correlation between the LND calculation methods and dosimetry. MCNP estimations appear closer to the dosimetry results for steady-state runs, as expected. MCNP values do deviate from dosimetry results by the same amount as the pulse runs, $\sim 0.4 \mathrm{E}-14$ [C/fission], but unlike the pulse results the steady results are a mix of over-estimation and under-estimation, with an nearperfect match with dosimetry for the $88.1 \mathrm{MJ}$ data.

\subsection{Overall Evaluation of Calibration Factor}

The results from both the pulse and steady-state experimental data can be combined and averaged in order to determine the proper value for the conversion factor $F$. In order to properly average the data, it should be weighted based on both consistent results and engineering judgement. First, as previously described, the MCNP data collected for the pulse runs appears to overestimate $F$. The experimental dosimetry results are regarded as much more reliable in practice at ACRR, so the pulsed MCNP data is ignored in the averaging. Second, the slight discrepancy between the average value of $F$ between the steady-state and pulse runs, $\sim 14 \%$, is possibly due to the steady-state runs. These runs required the reactor to ramp up to power beforehand and occurred over a longer period of time, so the dosimetry foils likely registered slightly more neutrons than those present over the experimental timeframe. Thus, averaging will be weighed slightly more towards the pulsed results from the CIC data by a factor of $1 / 10$.

After averaging and combining the experimental data, the final average value for the calibration factor $F$ is given by:

$$
F=3.26 \cdot 10^{-14}\left[\frac{C}{\text { fission }}\right] .
$$

With the data points having a standard deviation of $4.62 \%$. As for uncertainty, nickel foils possess about $4.3 \%$ and sulfur about $3.6 \%$. There are uncertainties for the values $G$ and $A$ of $1.5 \%$ and $1.6 \%$ respectively, and MCNP uncertainty depends on uncertainty in the neutron spectrum being modelled plus uncertainty in the reported energy for each run. The value stated above for $F$ is unique to the Photonis fission chamber that was used, and dependent on the detector's fill gas, geometry, and operating voltage difference.

Now that the parameters $F$ and $A$ are known for the Photonis fission chamber, researchers at ACRR can utilize Eq. (2) to relate the detector current to a neutron flux profile. This operation can be performed 


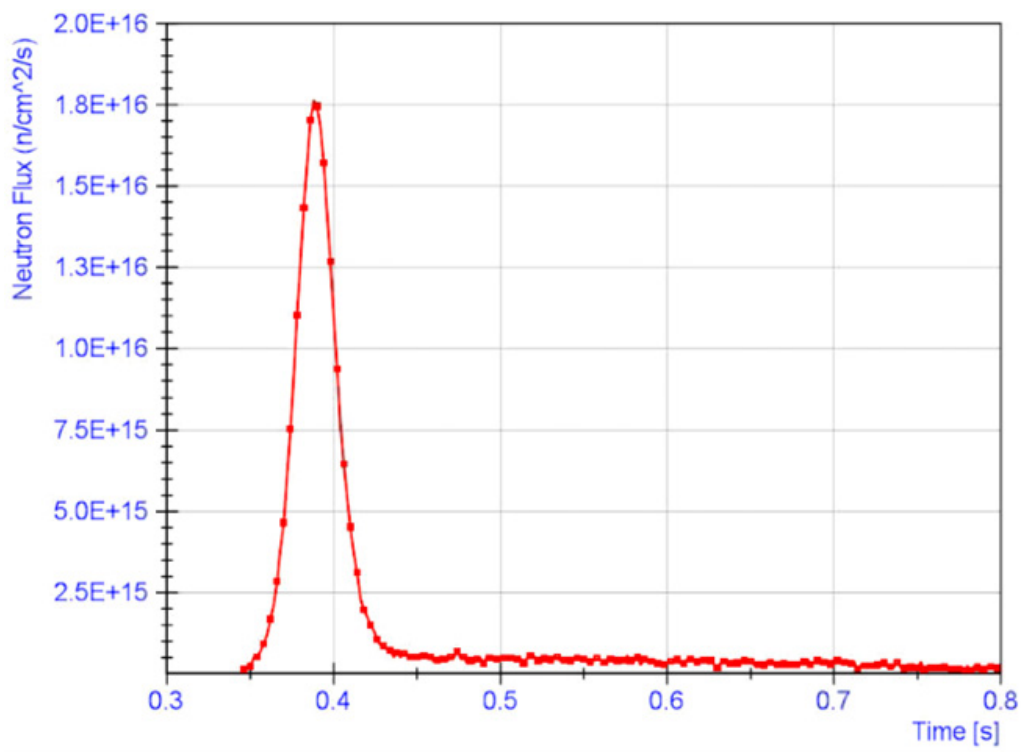

Figure 8. Neutron flux profile for run \#3 (50 MJ pulse).

for any well-characterized reactor environment for which $\overline{\Sigma_{f}}$ is known, and can be performed just a few minutes after data has been retrieved. For example, the fission chamber pulse data taken from Fig. 3 can now be directly converted to the following time dependent neutron flux profile.

The neutron fluence measured by integrating the fission chamber data from Fig. 8 was $6.75 \times 10^{14} \mathrm{n} / \mathrm{cm}^{2}$, which differed from the nickel and sulfur dosimetry fluences by about $3.9 \%$.

\section{Conclusions}

The new experimental calibration technique was successfully tested and implemented for the Photonis fission chamber for use at Sandia National Laboratories' ACRR facility. The calibration technique has allowed for the experimental determination of the calibration factor $F$ for the detector, representing the number of coulombs of charge produced per fission event for the chamber. The determined value of $F$, Eq. (7), has a standard deviation of the points of $4.62 \%$. When combined with known parameters of detector area $A$ and fission cross section $\overline{\Sigma_{f}}$, the current output of the fission chamber can be directly converted to a neutron flux profile as a function of time by Eq. (2), as demonstrated in Fig. 8. Thus, experimenters at the ACRR facility can now gather data on the neutron flux profile of future pulsed or steady-state experiments, just a few minutes after data collection. Experimenters will be able to immediately determine if the desired neutron fluence or flux was achieved for each ACRR experiment. In addition, the same calibration technique can be utilized for future fission chamber / compensating ion chamber pairs for use at the ACRR facility.

Future work in this area includes more extensive testing of the calibration technique at the ACRR facility. Having more data points for various energies is desirable to find an average $F$, especially towards the middle and upper limits of the ACRR's pulse capabilities. Also, the fission chamber should be tested in various other reactor environments, such as in PLG environments and free field environments. The calibration factor $F$ should be invariant of the reactor environment, while $\overline{\Sigma_{f}}$ is directly dependent on environment, and should be tested before the fission chamber's direct implementation at ACRR. Finally, more accurate MCNP models can always be implemented, and different dosimetry methods can be investigated. 


\section{References}

[1] G.F. Knoll, Radiation Detection and Measurement, John Wiley \& Sons, 2010, pp. 523-527

[2] J.T. Goorley, et al., Initial MCNP6 Release Overview - MCNP6 version 1.0, Los Alamos National Laboratory, report LA-UR-13-22934, April 24, 2013

[3] P.J. Griffin, J.G. Kelly, D.W. Vehar, Updated Neutron Spectrum Characterization of SNL Reactor Environments, Vol.1: Characterization, Sandia National Laboratories, Albuquerque, NM, SAND93-2554, April 1994

[4] K. R. DePriest, P. J. Cooper, and E. J. Parma. MCNP/MCNPX Model of the Annular Core Research Reactor. SAND Report, SAND2006-3067, Sandia National Laboratories, May 2006

[5] J. K. Shultis, R. E. Faw, Fundamentals of Nuclear Science and Engineering, Second Edition, Taylor and Francis Group, 2008, pp. 150-181

[6] E.J. Parma, T.J. Quirk, L. L. Lippert, P.J. Griffin, G.E. Naranjo, S.M. Luker, Radiation Characterization Summary: ACRR 44-inch Lead-Boron Bucket Located in Central Cavity on the 32-inch Pedestal at the Core Centerline (ACRR-LB44-CC-32-CC), SAND 2013-3406 